\title{
Intelligent Control Methods for Systems with Dispersed Generation
}

\author{
K. Tomsovic \\ School of Electrical Engineering and Computer Science \\ Washington State University, Pullman, WA \\ tomsovic@eecs.wsu.edu
}

\author{
Department of Electrical and Computer Engineering \\ Kumamoto University, Kumamoto, Japan \\ hiyama@eecs.kumamoto-u.ac.jp
}

\begin{abstract}
Recent technology improvements in small generators and energy storage devices have provided new opportunity for dispersed energy resources at the distribution level. At the same time, deregulation has encouraged a more decentralized approach to power delivery. This paper proposes some approaches to power system operations and control at the distribution level. A methodology for system security and associated operations is introduced. A simulation environment has been developed. Some results are presented showing the effectiveness of the new control methods.
\end{abstract}

Keywords: Dispersed energy resources, distribution system security, intelligent controls.

\section{INTRODUCTION}

The increasingly competitive nature of the electric utility industry, promises to transform the delivery of power and energy services. A number of distributed power generation technologies, including fuel cells, microturbines, and new energy storage devices, are currently available, offering a variety of performance characteristics for meeting user needs. Microturbines, available from several vendors [1-3], have been receiving particular attention due to the competitive pricing of natural gas, low installation costs and limited need for maintenance overhauls. In addition, new communications, primarily in the form of the internet, allow very low cost controls and a diversification of services. From the customer's perspective, distributed energy resources (DER) can offer improved service reliability, better economics and a reduced dependence on the local utility. From the utility's perspective, these units can help address a number of reliability and infrastructure problems.

The possibility of a significant penetration of DER in the near future suggests more focus must be placed on effective operations. The concept of a distributed composed of several loosely connected autonomous power systems (APS) or microgrids has been proposed [4]. In such a system, generator units will be responsible for "nearby" load and independently dispatched but still dependent on outside sources for normal operation. The units can supply various ancillary services, such as, load following [5] or voltage support, as well as energy. Other possible new services include time-of-use pricing, location and price dependent reliability, maintenance functions and reserve supplies.

Each APS may be characterized by different capacities, independence of operation and quality of service. For example, a residential community may desire a low capacity system with relatively frequent interruptions at a low cost. The currently available analysis tools provide no good methods for understanding the operation of such systems. As such, this paper poses some important problems in operating the power system with an APS. The emphasis here is on the strategies, or "intelligence," for the distributed subsystems to be operated effectively as a whole.

These interconnected subsystems are likely to be primarily radial in structure, that is, similar to the traditional distribution system, but with multiple sources. As such, analysis begins from traditional distribution system reliability analysis. Note in the extant power system, distribution circuits contribute over $90 \%$ towards customer outage and supply unavailability [6]. The wide exposure to harsh elements and the radial structure design, which accepts single point failures for lower cost, leads to this result. The expectation is that the new structure could greatly improve this performance under appropriate operation of the various subsystems.

In this paper, an overall framework for the security and control is discussed. A simulation has been developed for testing various structures and control philosophies. An example system using intelligent controls is introduced. This highlights the philosophy of the proposed framework.

\section{DISTRIBUTION SYSTEM OPERATIONS}

Power system reliability can be classified into two components: security and adequacy [7]. Adequacy is the static evaluation of a system's ability to supply the load. Security refers to the system's capability to withstand contingencies and still maintain service to all customers and respect all equipment limits. Traditionally, these have been analyzed as separate issues and it is quite possible to have a reliable but insecure system (say, in a system where critical contingencies are numerous but occur rarely) and vice versa. In general, adequacy is focused on planning while security is focused on operations. In the APS architecture, security can effectively enter distribution systems analysis for the first time.

\subsection{Distribution System Security}

A variety of reliability indices for a distribution system have been defined [8]. These indices can be divided into three categories: single load point indices, customer orientated indices, and load orientated indices. Single load point indices, which provide service reliability data from an 
individual customer viewpoint, are the average failure rate $\lambda_{s}$,

$$
\lambda_{s}=\sum_{i=1}^{n} \lambda_{i}
$$

where $n$ is the set of components whose failure results in an outage at the given load point $s$ and $\lambda_{i}$ is the failure rate of critical components within a section $i$.

Security in the APS will depend on: one, the exposure of the subsystem to disturbances upstream and downstream, and two, the ability of an islanded subsystem to supply load. The system level definition of security requires that no disturbance lead to a customer outage. That measure is not practical for a radial system even with multiple sources. Further, security is a system viewpoint of little relevance to an individual customer. The following definition is proposed that combines traditional reliability measures with security.

\section{A radial system is $\underline{n-1}$ secure if no single downstream fault will lead to an outage and $n+1$ secure if no fault between a loads section and the substation will lead to an outage.}

Thus, if protection is set correctly, most present distribution systems would be operated $n-1$ secure but only systems that can island to support generation could be $\mathbf{n}+\mathbf{1}$ secure. Note also that the system may become $n-1$ insecure if it relies on downstream generators to cover capacity limits.

For islanding then adequacy measures need to be redefined. Let $P_{i}$ be the maximum loading on a section $i$ and $P_{i}^{\max }$ the maximum generation in section $i$, if downstream from $i$

$$
\begin{aligned}
\sum_{i=1}^{m} P_{i}<\sum_{i=1}^{m} P_{i}^{\max },(1) \text { is modified to } \\
\lambda_{s}^{\prime}=\left(\sum_{i=1}^{n} \lambda_{i}\right) \cdot\left(\sum_{i=1}^{m} \lambda_{j}\right)
\end{aligned}
$$

where $m$ are components whose failure would cut off supply from the subsystem.

Operations for $n+1$ security is achieved by having sufficient downstream capacity. A different level of security could characterize each load point, i.e., customer, as is done with the customer oriented adequacy indices. Security will be of unquestionable importance for operations but should be related to reliability, that is, risk of outage, to be meaningful for most customers.

\subsection{Intelligent Controls}

The above areas of concern focus on development of new measures of reliability and new tools for designing systems based on these measures; however, there is an overriding difference in design process that cannot be neglected. The APS are not only independent in terms of operation but also in terms of business ownership. There will be a significant barrier to optimizing the performance if interests conflict among owners of the subsystems. Information necessary to optimize the overall reliability may not be available.

The question arises as to what decisions can be made on the basis of "local" information. For example, a controller that normally provides effective stabilization for some unit may be ineffective if another unit responds in a manner that reduces overall system damping. Each subsystem will necessarily operate under greater uncertainty. Accordingly, there must be guidelines to ensure adequate information to sustain a functioning system. Here, each subsystem is expected to act autonomously to control it's own operations but receive support from the substation.

In this uncertain environment, traditional centralized algorithms for design, operation and control are not adequate. Systems are needed that can observe system operation, determine the current mode of operation and subsequently select appropriate algorithms. This includes systems that will respond to market data as well as physical system parameters. In this vein, the authors propose an Intelligent Power Supply System (IPSS). The controls within the IPSS include fuzzy logic controls to ensure robust response given limited or no information on overall system dynamics. The authors have investigated fuzzy logic stabilization control and analyzed the stability of such systems in terms of general rule structure descriptions of systems rather than precise analytical models [e.g., 9]

\section{SIMULATION ENVIRONMENT}

Time domain simulations have been developed in the SIMULINK $^{\circledast}$ environment of the general purpose technical computing software MATLAB ${ }^{\oplus}$. Commercially available software tools are generally limited in their ability to perform the appropriate type of simulations. In the following, a control structure is developed based on the models employed.

\subsection{Network Models}

Detailed network models using time domain characteristics were developed. For reasons of efficiency, an unbalanced power flow algorithm was then developed based on [10] using standard phasor calculations. This load flow model was integrated with time domain models for use in operation studies. For efficient computations in time domain studies, the load flow calculations are conducted at larger time steps then the time integration. This is allowable since the voltage deviations between time integration steps are quite small. Using the previous time step voltage solution for initialization, convergence is usually within one forward and backward sweep, except if a large disturbance (e.g., switching) occurs. 


\subsection{Source Models}

At present, there are not standardized and widely accepted models for the different types of DER units, say, as one might find for large synchronous generators. Efforts are under way to address these modeling concerns [11]. For the purposes of this paper, generally accepted models are employed, where available, for the simulation. These models do not address all the practical control problems one may encounter but can demonstrate effectively the proposed control method.

Five different types of DER source models have been developed: small hydro units, microturbines, photovoltaic units, induction generators driven by a wind turbine and energy capacitor systems (ECS) for short-term storage. These models are briefly described in the following:

1. Mini hydro unit: Standard hydro unit models are used adjusted for the smaller capacity.

2. Microturbine: The high revolution speed type of unit is modeled. These fast rotations result in generally slow mechanical power output response. This is modeled here as a slow ramp rate with a single time constant.

3. Photovoltaic: A constant current source is assumed that is not controllable. A random model for solar variation is used.

4. Wind units: Turbines with asynchronous generator are represented as a non-linear function of the wind speed, which varies randomly. Control is limited to real power output variations that can be managed through control of the rotor pitch blades.

5. ECS: These are a new developed technology that, relative to batteries, has low energy storage but much longer lifetimes in terms of charge and discharge cycles. Costs have recently dropped dramatically. They have extremely fast response times, which are modeled as instantaneous here.

\subsection{Control and Communication Strategies}

The traditional load following and regulation functions used frequency deviation and tie-line mismatch to adjust generator set points [12]. The authors have proposed a similar concept for distribution systems that neglects frequency deviation [6]. Here, the feeder line flow is scheduled to a particular point on the distribution system and the control signal is taken to be the mismatch from the scheduled flow. The assumption is that this flow is measured locally and a communication link exists with any unit supplying the load following service. The obvious benefit of the proposed structure is simplicity and need for limited communications. In addition, the following observations are made:

- By neglecting frequency, concerns of interacting with system frequency regulation are minimized. The relatively low inertia of some smaller units coupled with the slow ramp up rates for other units (for example, in high speed microturbines or in fuel cells) makes them in appropriate for frequency regulation. Here, none of the units within the IPSS have governor speed-droop.

- ECS can still provide load following functions given sufficient capacity.

- The ECS can be controlled to damp oscillations from line faults so as to improve dynamic security. Those functions are not demonstrated in the numerical example presented here.

\section{SIMULATION RESULTS}

Numerical simulations are performed on the system shown in Fig. 1 that is based on a distribution feeder in the Kumamoto area of Japan. The nominal voltage level is $6.6 \mathrm{kV}$. The system includes a small hydro unit of $5 \mathrm{MW}$ near the substation. The IPSS is connected towards the end of the feeder near a highly variable load. The IPSS contains a solar cell, wind unit and microturbine for generation an ECS for storage. Only the microturbine and the ECS are actively controlled. For flow deviations, the ECS runs in continuous mode either charging or discharging to match load. There is also a neutral mode where the unit output current is zero. The ECS is restored to nominal charge after the disturbance has settled to within limits. The objective of the load following control is to respond to small load changes of short duration. Sustained deviations are eventually followed by the microturbine.

In reference to the proposed security framework, the system operates as both $n-1$ secure and $n+1$ secure at each load up to point A. Disturbances at the substation (response not shown here), or elsewhere along the feeder up, can be effectively damped by response of the ECS. For the variable load, faults beyond point $\mathrm{A}$, at either B, load Ld7 or line section C, lead to loss of service. The most securely operated points on the system are upstream from A. From a security stand point, the IPSS tends to benefit nearby upstream loads the most.

Two simulations cases are shown here. These results have been verified using a detailed analog simulation. In the first case, only the microturbine is controlled. The load increases for several seconds, returns to the initial state and then increases again. Fig. 2 and 3 shows the power inputs from the wind and solar units as well as response of the microturbine and ECS response. In Fig. 2, the response without the ECS is shown while Fig. 3 includes the ECS unit. The power flow at location A, the total real power output of the IPSS and the variable load are given by $P_{u p}, P_{i p}$ and $P_{l o}$, respectively. With the ECS, the load changes can very be tracked very accurately.

\section{CONCLUSIONS}

This paper discusses an approach for operating a distribution system with several DER units controlled for secure operations. The simple simulations here demonstrate the basic ideas of the concept. The ECS is integral to providing 
faster response needed to damp dynamic instabilities as well as respond to faster load fluctuations. The ideas presented here on security and operations of such a system are rudimentary. There remains much to be addressed in terms of the best overall operations philosophies given significant numbers of DER units.

\section{REFERENCES}

[1] S. Hamilton, "Fuel Cell-MTG Hybrid - The most Exciting Innovation in Power in the Next 10 Years" Proceedings of 1999 IEEE-PES Summer Meeting, Edmonton, Canada, July 1999, pp. 581-586.

[2] M. W. Davis, et al, "Microturbines - An Economics and Reliability Evaluation for commercial, Residential, and Remote Load Applications," PE-480-PWRS-0-1-1998, IEEE Transactions on Power Systems (in press).

[3] J. H. Watts, "Microturbine: A New Class of Gas Turbine Engines," NERC, GGTN News on-line, Vol. 39: 1999, No. 1.

[4] K. Tomsovic, "Issues of Reliability and Security in Distributed Autonomous Power Systems," Proceedings of the Workshop on Intelligent Distributed Autonomous Power Systems, Hakone, Japan, July 1998.

[5] S. Li, K. Tomsovic, and T. Hiyama, "Load Following Functions Using Distributed Energy Resources," Proceedings of the IEEE PES Summer Meeting, Seattle, July 2000.

[6] R.N. Allan, E.N. Dialynas and I.R. Homer, "Modeling and Evaluating the Reliability of Distribution Systems," IEEE Transactions on Power Apparatus Systems, Vol. PAS-98, No. 2, Nov./Dec. 1979, pp.436-444.

[7] R. Billinton and R.N. Allan, Reliability Evaluation of Power Systems, New York: Plenum Press, 1984, pp. 210-239.

[8] IEEE Working Group on System Design, "Trial Use Guide for Electric Power Distribution Reliability Indices," Report p1366, Draft \#14.

[9] K. Tomsovic, "Stability Analysis Methods of Fuzzy Logic Controls for Power Systems," Proceedings of the 1997 International Conference on Intelligent System Applications to Power Systems, Seoul, Korea, July 6-10,
1997, pp. 104-108.

[10]D. Shirmohammadi and H. W. Hong, "A Compensation-Based Power Flow Method for Weakly Meshed Distribution and Transmission Networks," IEEE Transactions on Power Systems, Vol. 3, No. 2, May 1988, pp. $753-762$.

[11]CIGRE TF 38.01.10, Modeling New Forms of Generation and Storage, Draft Report, June 2000.

[12] A.J. Wood and B.F. Wollenberg, Power Generation Operation and Control, John Wiley \& Sons, New York, 1996.

\section{BIOGRAPHIES}

Kevin Tomsovic received the B.S. from Michigan Tech. University, Houghton, in 1982, and the M.S. and Ph.D. degrees from University of Washington, Seattle, in 1984 and 1987, respectively, all in Electrical Engineering. Visiting university positions have included National Cheng Kung University, National Sun Yet-Sen University and the Royal Institute of Technology in Stockholm. Currently, he is an Associate Professor at Washington State University. His research interests include Intelligent Systems and optimization methodologies applied to various power systems problems. From 1999 to 2000, he held the Advanced Technology for Electrical Energy Chair at Kumamoto University in Japan.

Takashi Hiyama received his B.E., M.S., and Ph.D. all in Electrical Engineering from Kyoto University, in 1969 , 1971, and 1980, respectively. He joined Kumamoto University in 1971, and he has been a professor of Electrical Engineering and Computer Science since 1989. During the period of June 1985 through September 1986, he was at Clarkson University, and was involved with Power System Harmonics research. His current interests include Intelligent Control of Electric Power Systems using fuzzy logic control scheme and neural network, measurements and real time control of photovoltaic system, and harmonic analysis in distribution systems. $\mathrm{He}$ is a senior member of IEEE, and a member of SICE of Japan, and Japan Solar Energy Society.

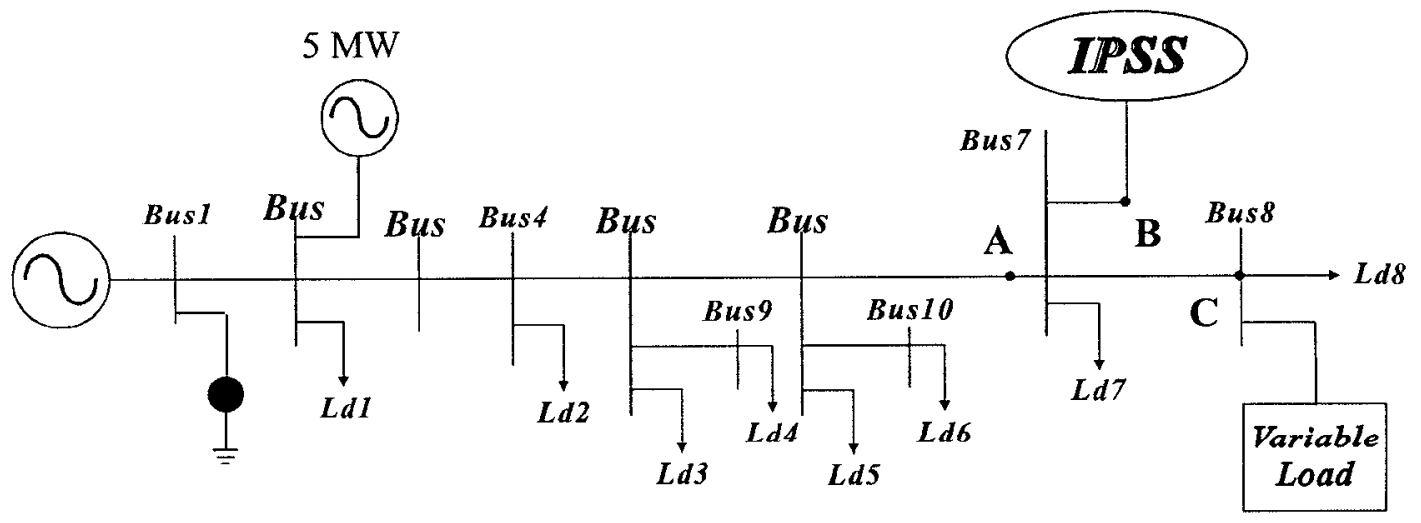

Fig. 1 Test System 

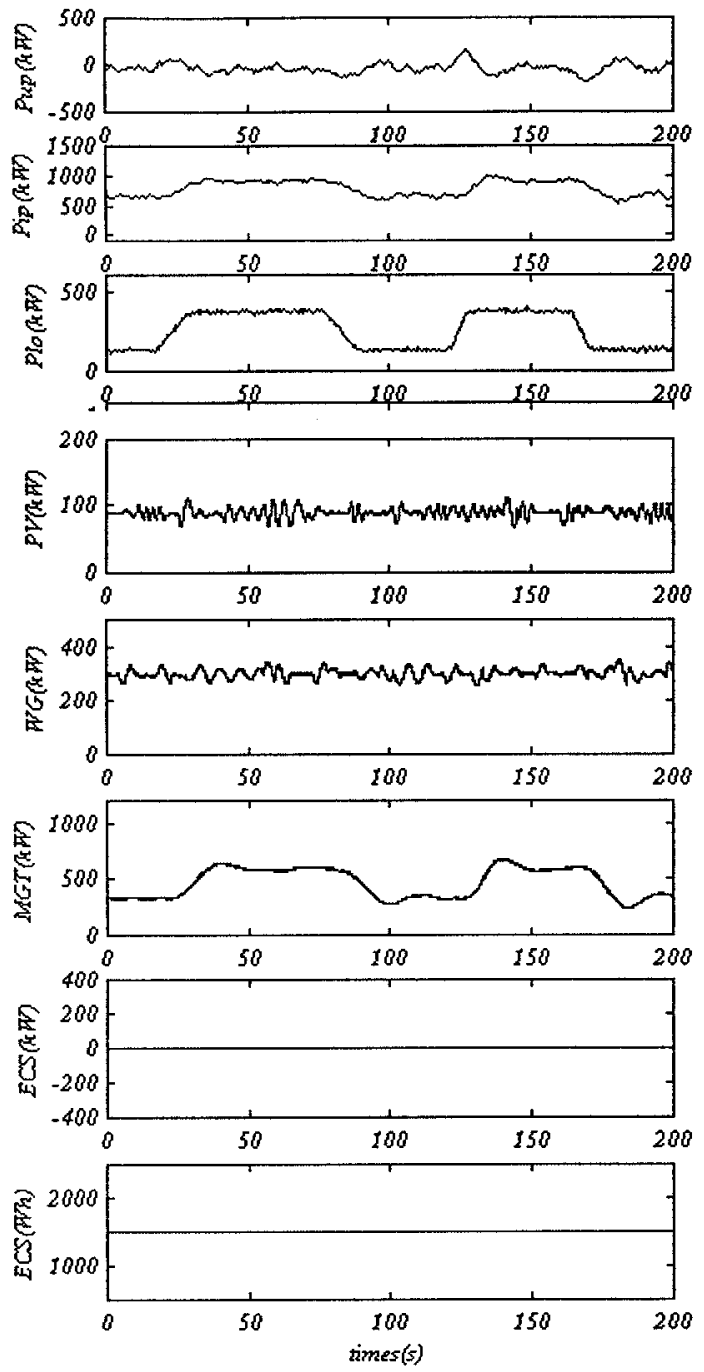

Fig. 2 Response to load change with no ECS
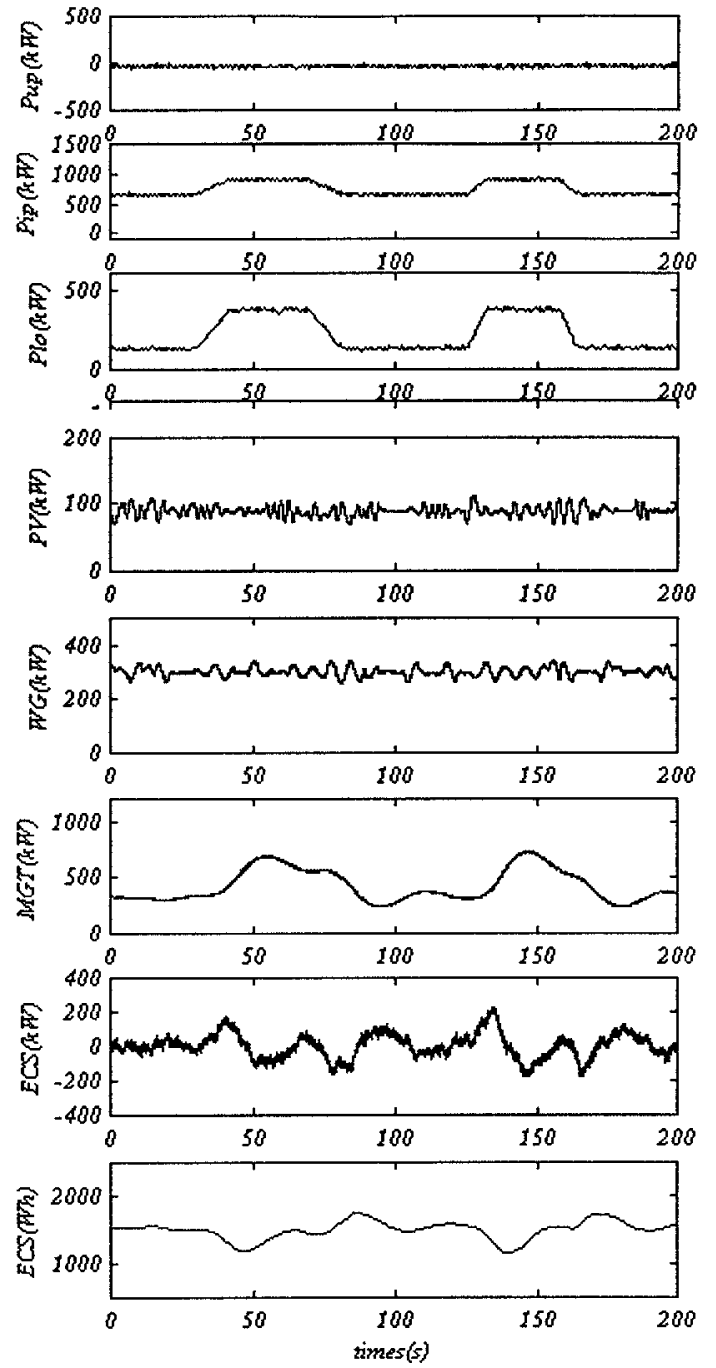

Fig. 3 Response to load change with ECS 